\title{
Les textes francophones malgaches sont-ils hybrides?
}

\author{
Dominique Ranaivoson
}

Université de Paris 13

Francophone literary production in Madagascar, although born out of a colonial context, has found its own voice in terms of the codes and themes it uses. It seeks to take its place in Francophone literature through comprehension based on a common language. However, the works written in French are informed by the cultural, social, spiritual and linguistic context of Madagascar. The resulting texts are full of allusions to prestigious literary genres, shared concerns and concepts whose comprehension is difficult for a readership which understands the words without understanding their cultural connotations. It is necessary to reflect on the specific task of the literary critic who may, whilst respecting the dynamics of a literary text, add annotations in the form of 'cultural translations'. The aim would be not to smother a body of work, which must be allowed to maintain its own nuances, but to allow better knowledge of the works and to make more effective the intercultural exchanges which are part of contemporary globalisation.

\section{Introduction}

Lorsque les Français annexent Madagascar en 1896, ils y instaurent rapidement et autoritairement une francisation de toutes les instances scolaires et politiques. Mais le champ littéraire n'était pas vide, toute une culture vivante en malgache était déjà développée. Les proverbes, les contes, les devinettes, les discours en formaient le matériau de base.

Durant les 64 années de colonisation, le réseau scolaire et la pression du système administratif ont encouragé la population à adopter le français comme langue de communication, le malgache demeurant la langue familiale, la langue de la prière, et la langue de création pour la plupart des écrivains, en particulier les poètes. Autrement dit, le malgache restait dans la sphère de l'intime et jouait un rôle important dans la mise à distance de l'étranger et la préservation d'une identité volontairement peu explicitée hors de la communauté.

Parallèlement à une littérature en malgache, une littérature francophone est née de ce système scolaire francisé. Ce dernier a en effet engendré une élite culturellement métissée, maîtrisant la langue et les références littéraires françaises sans avoir renoncé aux valeurs morales, sociales, esthétiques et spirituelles fondatrices de l'identité malgache. Citons, parmi les écrivains les plus remarquables, Jean-Joseph Rabearivelo (1902-1937), puis Flavien Ranaivo (1915-1999) et Jacques Rabemananjara (1913).

La production littéraire francophone contemporaine est l'héritière de ce double héritage, bien qu'elle soit passée, de 1975 à 1991, au travers du feu 
d'une époque hostile à la langue française pour des raisons idéologiques. Cette période fut marquée par une régression de la diffusion de la langue comme des idées occidentales, et une autarcie dans tous les domaines. La production francophone reprend maintenant de nouvelles audaces, et cherche à conquérir sa place au sein de la francophonie. ${ }^{1}$

Il nous faut examiner en quoi ces textes sont hybrides, ou métissés, comment ils sont construits et diffusés entre deux systèmes littéraires et sociaux, et comment une traduction d'un genre nouveau peut permettre de leur faire gagner une audience plus large au sein d'un monde culturel de plus en plus brassé. Jean-Marc Moura rappelle que l'hybridité est un concept spécifique à la critique postcoloniale élaboré par l'Indien Homi Kay Bhabha (Mongo-Mboussa 2003: 291-303), et que ce double héritage, cette double identité des textes, est en constante négociation à l'intérieur de chacun d'eux. Cette instabilité permanente en terme de situation par rapport à des références littéraires de l'une ou l'autre culture a des conséquences sur le statut de l'œuvre et l'élaboration d'une langue spécifique. Nous tenterons de voir si les textes malgaches sont dans ce cas, et quelles implications la critique peut en tirer quant à leur traduction.

\section{Quel entre-deux pour ces textes ?}

\subsection{De quels textes parlons-nous ?}

Les textes malgaches ne peuvent être résumés à quelques caractéristiques, leurs auteurs comme leurs genres étant variés. Nous distinguerons néanmoins quelques familles de pensée au sein desquelles une homogénéité est perceptible.

De nombreux Malgaches écrivent de la poésie en français, poésie marquée par les réminiscences de textes classiques assimilés durant la scolarité en même temps que des codes esthétiques et sociaux. Ces textes, quoique très nombreux, n'ont une importance que sociale, et ne cherchent pas de nouvelles pistes d'écriture, accordant toute leur attention au respect des conventions, elles-mêmes au service d'un contenu moralisateur. Ils retrouvent là la filiation avec les discours solennels malgaches appelés "kabarys", qui transmettent les valeurs à respecter.

D'autres auteurs, comme Charlotte Rafenomanjato, Christiane Ramanantsoa, Esther Nirina, écrivent des romans, des poèmes, des pièces de théâtre ancrés dans le contexte malgache et qui tendent à en exalter les valeurs, à encourager le lecteur à une réflexion sur la collectivité. La forme reste souple, mais ne s'écarte pas des modèles canoniques.

Enfin, d'autres francophones brandissent l'écriture comme une arme pointée contre la société, qu'ils décrivent comme entièrement viciée. Cette vision noire du monde peut être représentée par le fantastique comme dans Caprice-de-lune de Serge-Henri Rodin, ou la violence iconoclaste dans les gestes et les attitudes des personnages des ouvrages de Raharimanana, de 
Fota, et Jaomanoro, enfin par la révolte intérieure des personnages de la romancière Michèle Rakotoson.

Par delà leurs choix, ces différents auteurs sont tous puissamment enracinés dans la culture malgache, tout en s'exprimant en français, avec l'objectif affiché de toucher un public plus large que celui de Madagascar.

\subsection{Des textes français}

\subsubsection{Statut du français}

Ces textes sont écrits par des auteurs qui maîtrisent parfaitement le français depuis leur enfance tout en ayant le malgache comme langue maternelle, et choisissent de s'exprimer dans cette langue seconde pour différentes raisons.

Le français, qui a pu représenter la langue de l'occupant durant la colonisation (1896-1960), puis celle de la domination culturelle (19601972), est, depuis cette période de repli autarcique (1975-1991), associé au contraire à l'ouverture au monde. Le lien à renouer depuis l'île lointaine paraît à tous devoir passer par la langue, d'autant plus que les habitants ont pu se sentir oubliés quand le pays s'enfonçait dans la catastrophe économique. Ecrire en français, c'est donc s'ouvrir, échapper à l'enfermement insulaire, car, comme le dit le personnage d'une nouvelle de Fota: "Les îliens sur l'île. Ils rament sur l'île paradisiaque"'2 et Michèle Rakotoson, qui écrit en français après avoir écrit en malgache: "Une île, même aussi grande que Madagascar, c'est fermé” (Rakotoson 2002b).

Les auteurs pensent également avoir ainsi accès à un public plus large, qui les comprendra puisqu'il utilise la même langue. Il est ici important de souligner que la plupart des auteurs francophones n'ont pas conscience qu'une distance culturelle peut amoindrir la transparence de leur texte pour les francophones. Ils croient être de plain-pied dans une communauté où l'outil commun est suffisant pour une compréhension de tous par tous.

\subsubsection{Réticence}

Ces auteurs ont en face d'eux, à Madagascar, d'autres intellectuels tout à fait réticents à l'idée d'écrire dans une langue autre que celle de l'identité culturelle et nationale, allant jusqu'à trouver cette attitude signe de trahison, et réclamant une écriture de création en malgache, suivie d'une politique de traduction. Ce mouvement, sans être constitué en école, valorise avant tout la recherche identitaire, la réflexion anthropologique, le retour aux sources, avec, en toile de fond, une peur d'une invasion culturelle portée par les moyens économiques des grandes puissances. Pour ces chercheurs, la langue française ne peut rendre compte de la pensée malgache. Paradoxalement, ils semblent avoir confiance dans la traduction en français. 


\subsubsection{Métissage}

Tous les auteurs francophones sont conscients qu'ils entrent dans la langue d'écriture avec leur arrière-plan culturel. Ils ont le projet de transmettre celui-ci en investissant la forme. David Jaomanoro s' exprime ainsi: "La langue française est un no man's land. Je peux y pénétrer avec mes coffrets de masevy aux chevilles. Il y a un enrichissement mutuel entre une culture et une langue dans laquelle on s'exprime" (Jaomanoro 1991b: 31). Il est très loin le temps où écrire en français signifiait entrer dans le système de pensée et l'ensemble de règles données par le centre, Paris et ses normes en matières de langue et de valeurs.

Jeanine Rambeloson, enseignante de littérature à l'université de Tananarive, voit les jeunes écrivains hésiter puis faire le choix de leur langue d'écriture et conclut : "Ecrire en français, c'est un choix de civilisation; c'est adopter le métissage". 3

Et pourtant, dans la diffusion, en France ou au sein de la francophonie, personne ne prend en compte les conséquences de la réalité de ce métissage. L'immédiateté de la compréhension des mots semble permettre une lecture directe et une appréhension des différents niveaux de ces oeuvres. La mobilité sociale et culturelle de ces écrivains achève de convaincre qu'ils sont adaptés en tant que personnes, et que leurs textes sont aussi capables de circuler dans l'un ou l'autre champ. Quand la réception est timide ou quasi inexistante, la déception des auteurs les entraîne à mettre en cause la perspicacité du public.

Il nous semble que la question de la traduction surgit là, incontournable, pour ces textes en français, qui ne sont pas tout à fait français.

\subsection{Des textes malgaches}

\subsubsection{Formes et thèmes}

L'ensemble de la production actuelle malgache francophone traite de Madagascar: aucun texte ne se déroule ailleurs, ne sort des questions sociales, esthétiques, morales, politiques du pays. Bien sûr, une dimension poétique, fictive, onirique, accompagne, voire masque par instants cet arrièreplan, mais l'ignorer à la lecture ôte aux textes une bien grande part d'euxmêmes.

Dans la forme d'abord, la présence de personnages issus des contes traditionnels affirme un ancrage solide à des textes modernes. Nous retrouvons, dans des versions modernes et parfois inversées, les personnages du premier conte traditionnel, Ibonia, fixé depuis 1830. Ainsi, le jeune Ibonia assoiffé de liberté et d'aventures actualisées dans la pièce Ibonia de Michèle Rakotoson et Christiane Ramanantsoa (inédit, donné à Tananarive avec grand succès en 2000). Ainsi encore la sorcière Konantitra, femme profanatrice qui est la version féminine du gardien-esclave du conte, dans le 
roman Nour, 1947 de Raharimanana (2001). Ranoro, la fille des eaux d'un autre conte, est également en filigrane de Nour, 1947, au travers du nom de Nour, la femme morte qui se décompose dans l'eau. Dans le roman de Michèle Rakotoson, Henoy, fragments en écorce (1988), un homme cherche sa femme perdue à jamais. C'est d'un autre conte, celui du grand oiseau blanc Vorombe et de sa fille adoptive la princesse, que s'inspire, à la suite de Rabearivelo en 1935, Vololona Andriamoratsiresy avec sa pièce de théâtre Imaitsoanala (inédit, 1985). Charlotte Rafenomanjato présente au festival de Limoges en 1988 Le Prince de l'étang, qui est aussi une ré-appropriation d'une légende sur les vazimbas, ancêtres mythiques des Malgaches. Toutes ces légendes ne sont pas laissées dans leur contexte ancien et lointain, mais adaptées à la situation contemporaine, elles deviennent une façon de transmettre des réflexions audacieuses tout en restant dans la forme fidèle à la culture traditionnelle. Ce double jeu vise naturellement uniquement le public malgache, par ailleurs habitué aux messages esquissés et aux sous-entendus. Il est efficace dans la mesure où la fidélité des auteurs à la culture ne peut être remise en question, et que toutes leurs réflexions, même subversives, sur les questions brûlantes de l'actualité sociale, sont reçues comme telles.

Les traditions toujours en vigueur et parfois remises en cause sont également au cœur de nombreux ouvrages. Le bain des ossements royaux dans la mer qui a lieu tous les dix ans sur les côtes est le sujet central du roman Le Bain des reliques de Michèle Rakotoson (Paris, 1988). Le changement de linceul de l'ancêtre, qui rassemble toute sa descendance dans de grandes et coûteuses fêtes provoque des débats qui sont traités dans la pièce Impressions famadihana (inédite, donnée à Tananarive en 1999) et dans le conte Lamba (Madagascar, 2001) de Christiane Ramanantsoa. La croyance dans le destin tracé par les conjonctions astrales et dont on ne peut se délivrer que par des exorcismes structure le roman de Charlotte Rafenomanjato, Le pétale écarlate (Madagascar, 1990). La divination et ses dérives dans les campagnes est ironiquement représentée dans la pièce de David Jaomanoro Le dernier caïman (inédit, 1989).

Ensuite, la crise sociale, la dégradation des mœurs politiques avec le pouvoir despotique et la corruption, la paupérisation de tout le corps social sont des thèmes omniprésents de manière directe ou indirecte dans tous les textes de ces vingt dernières années.

Le thème de la mémoire, avec des allusions à l'esclavage dont les séquelles hantent la société malgache, et à la rébellion de 1947 dont l'interprétation n'est toujours pas consensuelle, reviennent dans les derniers ouvrages parus. Citons ce passage d'une pièce de théâtre inédite, Le mariage de la princesse Iangoria, où un père veut marier sa fille à un Européen. Il est fait allusion à la condition des descendants d'esclaves, officiellement affranchis depuis 1896, et dont le statut reste des plus marginaux:

Iangoria: O Ada, mon père, tu me prends pour une sauterelle qui ne rue pas dans la main. Je suis devenue une eau profonde qui donne le vertige; un rebut qui empoisonne le breuvage. Celui qui m'épouse écope sa vie durant. 
Vincent Ahmed: Si tu épouses le Belge, je t'assure que la mort elle-même baissera les bras devant ton opulence.

Ingoria: L'opulence entoure toujours une fille d'esclave, mais elle n'en profite pas. Penses-tu que mon mariage sera un sceptre entre tes doigts. Tes phalanges sauraient-elles encore blêmir autour d'un sceptre? D'ailleurs, le bâton n'élève pas la main qui le tient.

Les personnages principaux des romans Lalana (Rakotoson 2002a) et Nour, 1947 sont des descendants d'esclaves.

\title{
1.3.2. Genres littéraires traditionnels
}

Les genres littéraires traditionnels, qui forment la matière de la culture, et sont donc considérés comme 'typiquement malgaches', sont présents dans les textes contemporains sous forme de citations ou de modèles esthétiques. Les proverbes, substrat de toute la culture orale traditionnelle, et ces brefs poèmes nommés hain-teny émaillent, en français, la poésie de Maurice Ramarozaka (Tananarive, 1991: compte d'auteur, non paginé), au point de décontenancer celui qui ne les reconnaîtrait pas comme tels:

\author{
Euf d'alouette \\ Posé sur le chemin \\ Quiconque a des mains \\ Peut l'emporter \\ Las \\ Hérisson \\ Dans un buisson de framboisier \\ Les yeux l'atteignent \\ Mais les mains ne peuvent le saisir
}

Ce poème reproduit de manière voilée un discours sur la séduction, mais en avançant ainsi masqué derrière des citations, l'auteur peut se réapproprier ces thèmes et valoriser des paroles dont l'éclat est prestigieux, tout en montrant sa culture. Il reprend et combine ici dans chaque strophe un proverbe et un hain-teny. Dans la première, le proverbe parle de la disponibilité de la femme: "Des œufs d'alouette au bord du chemin; ce n'est pas moi qui les ai mis là, c'est la mère" et le hain-teny de celle de l'homme: "Si je dois être à vous/Je suis l'œuf d'alouette au bord du chemin". L'image du hérisson dans le framboisier est aussi une reprise: "Un hérisson dans les ronces: on le voit, mais il est difficile à prendre". La version poétique érotisée devient: "Me croyez-vous le tendrac dans le framboisier, Randriamatoa? Les yeux l'atteignent, les mains ne peuvent le saisir". ${ }^{4}$

Un tel poème ne choque ni ne déstabilise, le lecteur malgache y reconnaît au contraire la virtuosité dans la combinaison des citations qui est une des vertus du lettré. 
Le théâtre de Jaomanoro est également rempli de proverbes mis dans la bouche des personnages, souvent de J'ai marché dessus (1989): “Amère est la pierre qu'on m'a donné à lécher. Mes ancêtres m'ont-ils bénie à l'eau chaude? Mon chant se casse à peine entonné, comme le chant de la pintade".

Les hain-teny sont inclus, traduits, et reconnus, dans les poèmes de Vololona Picard (De jaspe et de sang, La Réunion, 1998), la poésie française de Dox (Chants capricorniens, Madagascar, 1994), la pièce Impressions famadihana, déjà citée. Les images traditionnelles et fortement symboliques en malgache, comme la valeur polysémique du bleu, le code rattaché au lait et au miel, se retrouvent dans la poésie d'Esther Nirina (Rien que lune, La Réunion, 1998). Le théâtre paysan dit hira gasy est la référence sous-jacente aux pièces de Michèle Rakotoson La maison morte et Un jour, ma mémoire (1991). Toutes ces citations fonctionnent comme des signaux qui garantiraient aux yeux du lecteur malgache, plein de méfiance devant cette production en français, une identité intacte parce qu'enracinée dans cet héritage. La présence de celui-ci donne également du prestige aux auteurs, qui manifestent ainsi leur érudition, dans la lignée des orateurs traditionnels capables de citer de nombreuses références en les contextualisant.

Cette accumulation pourrait faire croire que ces textes seraient opaques à qui n'aurait pas vécu à Madagascar. Sans atteindre cette limite à la compréhension, il nous faut nous interroger sur les conséquences de cette interpénétration entre langue et culture.

\subsection{Des textes hybrides}

Ecrite dans une langue commune à des aires culturelles très contrastées, la littérature francophone malgache contient en elle-même plusieurs ferments de contradictions.

Adossée à un corpus prestigieux de contes, proverbes, poésies, théâtre, elle y puise une intertextualité qui fait sa richesse pour ceux qui savent en discerner la présence, mais tisse une certaine opacité pour les autres. Nourrie dans ses thèmes et ses revendications par la situation sociale complexe, elle aborde quantité de questions étrangères aux autres sociétés (bien que certaines, comme le pouvoir, la corruption et l'injustice soient hélas connues de tous). La révolte toujours voilée, la violence apparemment sans objet, les dénonciations faites par allusions, peuvent déstabiliser un lecteur incapable de décrypter ces signes de désespoir:

Les gens comme lui restaient dans la basse ville, descendants d'esclaves issus des marges du royaume. Ils étaient les valala mpiandry fasana, les criquets gardiens du tombeau, ceux qui étaient destinés à rester là-bas, dans les campagnes et les rizières, les pieds dans la boue et les mains dans la gadoue, pour pérenniser une terre qui de toute façon ne leur appartiendrait jamais. (Rakotoson 2002a: 41) 
Les thèmes des séquelles de l'esclavage, de la répartition sociale de l'espace urbain, de la situation foncière des campagnes, thèmes connus quoique jamais abordés de front, sont présents dans cette simple phrase, audacieuse pour le lectorat malgache.

Marquée par le statut individualisé de l'écrivain occidental, cette production déconcerte aussi le public malgache en ignorant les contraintes tacites de correction, en osant aborder des sujets délicats, en s'écartant du rôle traditionnellement assigné aux écrivains, chargés de représenter leur communauté et de lui apporter un message constructif. Cette individuation de l'écrivain, dans le choix des thèmes comme dans la recherche de nouvelles formes stylistiques, est la plus grande marque de la distance prise par certains francophones.

Mais ces deux appartenances culturelles ne sont pas figées en un 'entre-deux' bien défini ni équitable. De manière tout à fait inconsciente pour les auteurs, ce métissage est en constante variation, chacun des pôles pesant davantage selon les thèmes abordés, la situation, l'émotion de l'instant, le lieu de l'écriture. Nous retrouvons l'élément variable que Jean-Marc Moura nommait 'négociation'. Tous les éléments de l'hybridité nous semblent rassemblés.

Ainsi, l'écrivain malgache francophone se trouve-t-il à la lisière de deux mondes, deux logiques, deux systèmes de communication. La circulation matériellement facile des textes permet-elle de faire l'économie d'une réflexion sur les limites, et les éventuelles interfaces nécessaires à une meilleure compréhension de ces productions dans l'une et l'autre de ses aires de diffusion?

\section{Quelle traduction?}

\subsection{La langue}

\subsubsection{Termes malgaches}

Plusieurs auteurs ressentent la nécessité d'employer un terme malgache pour désigner des objets ou des concepts qui n'existent pas en français. Ainsi, les œuvres de Jaomanoro, les recueils de Vololona Picard, d'Esther Nirina, de Hery Mahavanona, comportent-ils un glossaire en fin de volume. Michèle Rakotoson insère des chansons ou des prières en malgache, et les traduit en général au sein même du texte, lui donnant ainsi deux niveaux de lecture, cherchant à expliquer la puissance de la charge émotive contenue dans ces citations (Ranaivoson 2003a; 2003c). D'autres auteurs n'emploient aucun terme malgache, mais jouent sur la toponymie, en traduisant les noms de lieu en français comme Serge-Henri Rodin, ce qui crée un effet d'énigme pour celui qui cherche à retrouver les vrais noms ainsi cachés: 'L'arrivée au grand port Qui-rend-Vigoureux fut des plus désagréables[...] Aucune navette ne partait immédiatement pour la ville Où-il-y-a-beaucoup-de-crocodiles... " (Rodin 2000). 
Sont désignées ici les villes de Majunga et de Marovoay, énigmes simples pour les Malgaches, mais qui entretiennent une lecture qui tient du décryptage. Voulant franciser au maximum ses textes, l'auteur en arrive à les rendre déchiffrables sur le plan lexical pour le francophone, mais ils ont perdu tout enracinement, ce qui leur enlève leur sens, les 'délocalise', plutôt les 'déterritorialise' et les fait accéder ainsi au statut universel de fable.

D'autres jouent sur les énumérations des noms malgaches, basant la puissance du texte sur les connotations de ceux-ci. Les montagnes et les noms des villages jouent ce rôle chez Ramarozaka:

Et, ces larmes

Larmes d'Ambatomiatendro

Larmes de l'Ankaratra

Qui languissent de s'unir

Mais que sépare la Grande-Indifférente

Il tonne là-bas du côté de l'Ankaratra

(Ranarozaka 1991: pas paginé)

Esther Nirina (1998) évoque ces mêmes montagnes et fleuves qui ne sont que les métonymies du pays tout entier: "Chaque note/De mes automnes/ Module/Dans leur diapason/Angavo.... Ankaratra/ Managareza".

Hery Mahavanona égrène au contraire les lieux oubliés qui symbolisent son identité à reconquérir: “j’évoquais vos noms/comme une prière/Madiorano, Tolongoina, Amboanjobe, Fenomby". ${ }^{5}$

Le jeu des noms est ici tout entier basé sur leurs sonorités, qui échappent à celui qui ne sait les prononcer à la malgache, et parcourt ces syllabes étranges sans émotion particulière, si ce n'est de sentir l'étrangeté qui le sépare de cette culture. Paradoxalement, l'évocation de lieux proches a provoqué la distance.

\subsection{2. 'Hétérolinguisme' et 'interlangue'}

La question de la correction de la langue se pose en particulier dans les textes de Jaomanoro, qui donne presque systématiquement la parole à un narrateur s'exprimant dans un français calqué sur les structures du malgache, largement émaillé d'expressions locales, ou de mots comoriens dans les textes sur Mayotte. Ici, la femme du Petit os s'adresse à son ancienne rivale devant la dépouille de celle-ci:

Maintenant, je n'ai plus de restes contre toi. Pour moi, les restes, ce ne sont pas les quartiers de viande que l'on jette après l'enterrement: c'est du gaspillage. On ne mange des bœufs que pendant les funérailles, et après on jette les restes aux chiens! Inconscience. Les restes, pour moi, ce sont les cœurs que l'on garde contre autrui. (1991a: 36)

Jean-Marc Moura qualifie ces procédés d' 'hétérolinguisme' (1999: 73-91), K.Vogel d' 'interlangue'. Il s'agit de procédés stylistiques qui permettent de 
rendre compte devant un lecteur souvent décontenancé ou charmé d'un possible fonctionnement complètement différent de cette langue française qu'il croyait maîtriser avec ses normes annoncées comme universelles. La doxa avait fait croire au caractère univoque de celles-ci. Cette languemétisse vient contredire de fait toute autorité unique, et confirmer qu'en matière de langue, l'usage et la communauté sont plus puissants que tout centre prestigieux. Cette littérature comprend donc des ferments de revendications d'une périphérie qui a assimilé les outils à sa manière, dans son contexte et selon son imaginaire, pour rendre compte de son environnement propre.

Ces incursions et combinaisons de la langue malgache dans ces textes francophones nous confirment que ceux-ci sont les fruits intimes d'un métissage entre un imaginaire, une culture, et deux langues. Le code linguistique français, apparemment adopté avec soumission, est en réalité doublé de plusieurs autres codes, sociaux, esthétiques, affectifs, tous faisant référence au système malgache.

La poète Esther Nirina résume cette situation dans un poème où elle emploie le mot 'hybride', elle qui est entre cet 'ici' français et ce 'là-bas' malgache, ou inversement:
A mi-chemin du pont
Où vacille l'image de l'autre
Trouvez-moi le mot
Qui mène à terme la traversée
Avoir ainsi pour premier jet
L'hybride né du langage
D'ici et de là-bas
Les cicatices
D'où écoulement du sang
Ré-inscrit
La rose
(Nirina 1998: 27)

Cette interculturalité enrichit considérablement les textes en ce qu'elle leur donne une multiplicité de sens, mais elle les rend opaques à ceux qui ne décodent pas les différentes symboliques. La question se pose d'une intervention d'un tiers qui tendrait à ouvrir pour le lecteur des pistes d'interprétations.

\subsection{Transposition}

Le poète Rabearivelo, bilingue et surtout passionnément attaché aux deux cultures, s'est essayé à donner des traductions qui rendent compte des cultures et de l'imaginaire des langues. Il a été dit 'passeur de langue' pour ces tentatives de superposition des deux mondes auxquels il a tenté d'appartenir complètement et conjointement ${ }^{6}$. Flavien Ranaivo a, après Jean Paulhan et 
lui, traduit des hain-teny, tentant lui aussi de traduire le cœur même de la sensibilité malgache ${ }^{7}$.

La réception de ces textes, enthousiaste chez les intellectuels français autour de Paulhan, a permis la découverte de ce genre inconnu en Europe. Mais la traduction rend-elle l'émotion de l'ellipse, l'admiration provoquée par l'énigme des questions ou la pirouette des fins? Les noms propres très longs en malgache et qui sont constitués d'une agglutination formant les caractéristiques du personnages sont-ils aussi évocateurs quand ils sont traduits littéralement, devenant "Celle-dont-les-pas-résonnent-des-jours", "Celui-qui-ne-craint-pas-le-châtiment-du-mal" (Rabearivelo 1974: 25). Le préfacier à l'une des éditions de ces poèmes traduits par Rabearivelo, luimême poète malgache, Lucien Michel-Andrianarahinjaka, parle de 'transposition' et non de 'traduction', mettant en évidence l'effort de Rabearivelo pour faire pénétrer dans l'univers malgache, au détriment de la fidélité au texte. Jean-Marc Moura parle, de "subversion expressive de la langue française" (Moura 1999: 81). Il faut avouer que celle-ci, bien que française, reste extrêmement mystérieuse pour un lecteur non averti des codes employés:

Pauvres nénuphars bleus: toute l'année ils ont des larmes jusqu'au cou! Brins d'herbes d'eaux, brins de jonc de mares charriés par les pirogues, abritezmoi: je suis si malheureuse! Volez pour moi un peu d'amour: je suis à un autre! Votre femme, aimez-la; moi, ne m'abandonnez pas! Qui n'a pas de piment, il n'éprouve pas de volupté en mangeant; qui a perdu son piège à poissons n'aura pas de friture. Et moi, si je vous perds, je perdrai mon plus proche parent! (Rabearivelo 1974)

Faut-il laisser au texte une part d'opacité afin qu'il garde cette empreinte d'hybridité qui fait son identité, et son charme? Où doit commencer l'éclaircissement?

\subsection{L'éclosion du texte seul}

Pour les textes francophones, il est clair que toute insertion de mots, d'expressions ou de citations en malgache est voulue, maîtrisée, calculée. Il serait abusif qu'un quelconque traducteur se surajoute en ces lieux.

Mais un appareil critique ne serait-il pas nécessaire pour dire la place du Mont l'Ankaratra, tant de fois cité dans la poésie, la signification sociale du silence qui entoure telle question qui serpente au long de telle œuvre, la symbolique d'une expression, la raison de telle révolte ou de telle émotion devant une situation ou un lieu précis, le statut social de ce personnage?

Faut-il expliquer le sens des traditions dont la transgression ou l'incompréhension déclenchent le drame dans plusieurs œuvres?

Nous le pensons, tout en gardant une autre priorité, celle de l'éclosion du texte seul. L'écriture est liberté, liberté de dire ou de cacher, d'expliquer ou de voiler. Celle-ci ne serait-elle pas dévoyée si une introduction ou des 
notes venaient mettre à plat ce qui est déployé en images, demi-mots, symboles, constructions narratives?

La lecture est, elle aussi, un acte personnel et libre, où le jeu de l'interprétation excite l'imagination, pose des hypothèses au fur et à mesure qu'avance le texte. Bien que le déroulement syntaxique soit linéaire, l'acte de construction du sens qui se joue chez le lecteur use des va-et-vient à l'intérieur même du texte. Eclaircir chaque difficulté par des notes explicatives immédiates n'aboutirait-il pas à tuer cette circulation nécessaire à une lecture d'autant plus active que le texte résiste?

L'accumulation des explications fragmentées des glossaires placés en fin de volume nous paraît une mesure qui garde la fluidité au texte, mais ne rend pas compte de l'imbrication des éléments présents en une vie complexe et particulière.

Une introduction qui décrit brièvement l'arrière-plan culturel des textes nous paraît beaucoup plus adaptée. Elle retarde l'immersion dans le texte, mais y prépare le lecteur qui est en quelque sorte mis en condition, muni de quelques outils d'interprétations, de quelques codes grâce auxquels il entrera plus vite dans l'épaisseur et la polysémie du texte. L'amateur de dépaysement enjambera ce qu'il prendra pour un encombrant préambule, mais y reviendra sans doute après une première lecture qu'il aura désirée "naïve" et où il aura senti les limites de sa propre panoplie interprétative.

Dans le même respect du texte comme du lecteur, quelques analyses placées en notes aideraient à donner leur portée exacte aux éléments présents. Ces informations seront succinctes, et ne sauraient fermer l'éventail des interprétations et ainsi réduire le sens, mais simplement apporteraient des éléments neutres, utiles pour orienter celui-ci dans la bonne direction.

Maryse Condé, Antillaise écrivant en français et vivant aux EtatsUnis, rappelle que 'l'auteur n'est qu'un traducteur. Traducteur de son Moi. De son imaginaire. Et de sa réalité" (Kandé 1999: 210). Ecriture singulière car individuelle dans les sociétés occidentales, Moi particulier dans un système où l'écriture est une création personnelle, mais imaginaire et réalité communs, tout du moins en partie, à une communauté culturelle à laquelle n'appartient pas le lecteur francophone (occidental par exemple). Si la confiance dans la langue employée fait croire à une compréhension immédiate, les obstacles de cette réalité non partagée viennent immédiatement dresser des barrières à l'intelligibilité du texte. Certes, il en est qui aiment et cultivent celle-ci jusqu'à en faire un dogme. L'altérité devient alors une fierté, la distance une justification à la revendication. Mais il nous semble que ces littératures issues de la périphérie sont écrites pour être reçues par un lectorat qui aime et cultive cette ambivalence entre désir de comprendre, donc de maîtriser intellectuellement, et fascination pour un 'ailleurs' que l'on regarde sans y pénétrer, dont on ne garde que les aspects exotiques grâce à une mise à distance protectrice. La part d'incompréhensible jouerait là son rôle de fossé protecteur contre tout danger d'empathie sans retour.

Ce serait sans doute contraindre par trop l'auteur que de lui demander d'être plus explicite dans cette traduction de son Moi. C'est l'objet même de 
son écriture toute entière qui tend vers ce but, et c'est la joie et la liberté de la création littéraire de choisir des chemins détournés, escarpés, étroits, elliptiques, pour y parvenir.

\section{Conclusion}

Cette littérature francophone, née loin du centre de la francophonie et des lieux d'autorité en matière de langue, puise largement son inspiration dans un contexte social et une culture malgache originaux. La langue employée se trouve enrichie de termes particuliers, de tournures venues du malgache. Une intertextualité tisse des liens constants avec la littérature traditionnelle. Il s'ensuit une langue écrite en français, mais si fortement nourrie de la culture malgache que l'on peut la qualifier d'interlangue. La littérature n'est plus uniquement malgache, n'est pas française, mais authentiquement interculturelle, hybride.

Cette situation d' 'entre-deux' crée des opacités de part et d'autre des publics, qui proviennent d'aires culturelles homogènes (ou du moins plus homogènes) et ne peuvent décrypter les connotations propres à un milieu qui n'est pas le leur sans que des indications soient données en marge du texte.

Il nous semble que le travail de décodage, d'explicitation, de traduction culturelle, est du ressort du critique, qui accompagne le texte, l'enveloppe sans l'étouffer, l'explique sans tout dévoiler, l'introduit sans le dénuder. Il est à l'interface de deux systèmes, de deux imaginaires, devant une langue qui est en réalité double.

Si sa présence reste secondaire, humble, il peut devenir ce 'traducteur culturel' qui permettra aux textes lointains de devenir proches, qui saura respecter cette irréductible altérité fondatrice pour chacun de son identité, qui permettra à la mondialisation de ne pas faire croire à une uniformisation des hommes. La littérature pourra alors être ce lieu où se vit une authentique interculturalité.

\section{Bibliographie}

\section{Sources primaires}

Combe, Dominique (1995). Poétiques francophones. Paris: Hachette.

Dox (1994). Chants capricorniens. Tananarive: Centre culturel Albert Camus.

Houlder (1960). Ohabolana. Tananarive: Imprimerie luthérienne.

Jaomanoro, David (1989). La Retraite. Belgique (Carnières, Editions Promotion du Théâtre): Lansman.

Jaomanoro, David (1991a). "Le petit os.” Nouvelles francophones. Printemps culturel du Valenciennois.

Jaomanoro, David (1994). "Les funérailles d'un cochon." Funérailles d'un cochon et 13 autres nouvelles. Paris: Sépia. 
Jaomanoro, David (2000). “Jaombilo.” Nouvelles francophones. Paris: Acoria. Ligier, Françoise (éd.) (1991). Théâtre Sud 3. Paris: Karthala.

Mahavanona, Hery (1999). Urgence d'écriture. La Réunion: Grand Océan.

Nirina, Esther (1998). Rien que lune. La Réunion: Grand Océan.

Paulhan, Jean (1991). Hain-teny merina. Tananarive: Foi et Justice.

Picard, Vololona (1998) De jaspe et de sang. La Réunion: Grand Océan.

Rabearivelo, Jean-Joseph (1974). Vieilles chansons des pays d'Imerina. Tananarive: Madprint.

Rabemananjara, Jacques (1978). CEuvres complètes, poésie. Paris: Présence africaine.

Rafenomanjato, Charlotte (1990). Le pétale écarlate. Tananarive: Société malgache d'édition.

Rafenomanjato, Charlotte (1994). Le cinquième sceau. Paris: L'Harmattan.

Rafenomanjato, Charlotte (2003). Sang pour sang, vie pour vie. Tananarive: SME.

Raharimanana (1996). Lucarne. Paris: Le Serpent à plumes.

Raharimanana (1998). Rêves sous le linceul. Paris: Le Serpent à plumes.

Raharimanana (2001). Nour, 1947. Paris: Le Serpent à plumes.

Rakotoson, Michèle (1988). Le bain des reliques. Paris: Karthala.

Rakotoson, Michèle (1998). Henoy, fragments en écorce. Belgique (Avin/Hainaut): Luce Wilquin.

Rakotoson, Michèle (2002a). Lalana. Paris: L'aube.

Ramanantsoa, Christiane (2001). Lamba. Tananarive: Tsipika.

Ramarosoa, Liliane (1994). Anthologie de la littérature malgache d'expression française des années 80. Paris: L'Harmattan.

Ramarozaka, Maurice (1991). Madécasseries. Tananarive: compte d'auteur.

Ranaivo, Flavien (1975). Poèmes, hain-teny. Paris: Publications orientalistes de France.

Rodin, Serge-Henri (2000). Caprice-de-la-lune. La Réunion: Grand Océan.

\section{Sources secondaires}

Christiane Albert (éd.) (1999). Francophonie et identités culturelles. Paris: Karthala. Jaomanoro, David (1991b). "Entretien". Théâtre Sud 3, 31.

Kandé, Sylvie (éd.) (1999). Discours sur le métissage, identités métisses. Paris: L'Harmattan.

Mongo-Mboussa, Boniface (éd.) (2000). Africultures. Postcolonialisme: Inventaire et débats 28 .

Mongo-Mboussa, Boniface (2003). Désir d'Afrique. Paris: Gallimard.

Moura, Jean-Marc (1999). Littératures francophones et théorie postcoloniale. Paris: Presses universitaires de France, 'Ecritures francophones'.

Rakotoson, Michèle (2002b). "Entretien". Tribune, Juillet 9.

Ranaivoson, Dominique (2002). "Violence inattendue dans la littérature malgache contemporaine". Notre Librairie 148, 26-30.

Ranaivoson, Dominique (2003a). "Le sens du surgissement de la langue maternelle dans le texte francophone: l'exemple des romans malgaches de Michèle Rakotoson". Mots Pluriels 23. Traduire le vécu: l'autobiographie entre langues et cultures, revue internationale en ligne, University of Western Australia, Nedlands, Australie.

Ranaivoson, Dominique (2003b). "David Jaomanoro, un écrivain malgache francophone original". Notre Librairie 150, 92-97.

Ranaivoson, Dominque (2003c). "La chanson traditionnelle comme gage de stabili- 
té dans les romans malgaches de Michèle Rakotoson". Actes du colloque Chansons en mémoire, mémoire en chansons, Saint-Hermine (Vendée), Paris 4 - CNRS.

Ranaivoson, Dominique (en publication). La littérature francophone à Madagascar. Paris: Karthala.

Toumson, Roger (1998). Mythologie du métissage. Paris: Presses universitaires de France, 'Ecritures francophones'.

Vérin, Pierre (1998). "Le français et les langues régionales de l'Océan Indien occidental". Langues et Pouvoir de l'Afrique du Nord à l'Extrême-Orient. Paris: Edisud, 83-93.

${ }^{1}$ Voir l'article de Pierre Vérin cité dans la bibliographie.

2 Jean-Claude Fota, L'Escale, nouvelle inédite, 3.

${ }^{3}$ Entretien personnel, Tananarive, 1999.

${ }^{4}$ Les proverbes sont répertoriés par Houlder, Ohabolana, Tananarive, 1960. Nous citons les $\mathrm{n}^{\circ}$ s 1042 et 1213. Les hain-teny sont cités par Paulhan, Hain-teny merina, Tananarive, 1991, ${ }^{\circ} 7$, p.63 et $\mathrm{n}^{\circ} 10, \mathrm{p} .65$. Le tendrac est une sorte de hérisson.

${ }^{5}$ Hery Mahavanona, poème "Evocation du pays tanala", Urgence d'écriture, Grand Océan, La Réunion, 28.

${ }^{6}$ Son suicide en 1937 est le signe de l'échec de cette tentative de totale double identité qui ne lui a pas été reconnue.

${ }^{7}$ Jean Paulhan, Hain-tenys merina, 1ère édition à Paris, Geuthner, 1913, puis ré-édition par Gallimard en 1939 et à Tananarive en bilingue par Foi et Justice, 1991, que nous utilisons ici. 